\title{
On Some Bounds for the Exponential Integral Function
}

\author{
Kwara Nantomah \\ Department of Mathematics, School of Mathematical Sciences, \\ C. K. Tedam University of Technology and Applied Sciences, \\ P. O. Box 24, Navrongo, Upper-East Region, Ghana. \\ Correspondence to: knantomah@cktutas.edu.gh
}

\begin{abstract}
In 1934, Hopf established an elegant inequality bounding the exponential integral function. In 1959, Gautschi established an improvement of Hopf's results. In 1969, Luke also established two inequalities with each improving Hopf's results. In 1997, Alzer also established another improvement of Hopf's results. In this paper, we provide two new proofs of Luke's first inequality and as an application of this inequality, we provide a new proof and a generalization of Gautschi's results. Furthermore, we establish some inequalities which are analogous to Luke's second inequality and Alzer's inequality. The techniques adopted in proving our results are simple and straightforward.
\end{abstract}

Keywords: Exponential integral function, Incomplete gamma function, Bounds, Inequality

DOI: https://doi.org/10.3126/jnms.v4i2.41463

\section{Introduction}

The classical exponential integral function is usually defined as [1, p. 228]

$$
\begin{aligned}
E(z) & =\int_{z}^{\infty} \frac{e^{-r}}{r} d r \\
& =\int_{1}^{\infty} \frac{e^{-z r}}{r} d r \\
& =\Gamma(0, z)
\end{aligned}
$$

for $z>0$ where $\Gamma(v, z)$ is the upper (or complementary) incomplete gamma function defined as

$$
\Gamma(v, z)=\int_{z}^{\infty} r^{v-1} e^{-r} d r .
$$

It satisfies the properties

$$
\begin{gathered}
E^{\prime}(z)=-\frac{e^{-z}}{z} \\
E^{\prime \prime}(z)=\frac{e^{-z}}{z}+\frac{e^{-z}}{z^{2}}=-\left(1+\frac{1}{z}\right) E^{\prime}(z),
\end{gathered}
$$

among others. For more properties of the function, one may refer to [1, 10, 14. This special function is often applied in areas such as astrophysics, neutron physics, quantum chemistry and engineering. As a result of its important roles, it has been studied in various ways. For instance, see [3, 4, 11, 12, 13, 15, 16, 17, 18,

In 1934, Hopf [7, p. 26] established the inequality

$$
\frac{z}{z+1}<z e^{z} E(z)<1 \quad \text { for } \quad z>0
$$

In 1959, Gautschi [5] gave an improvement of 4 by establishing that

$$
\frac{1}{2} \ln \left(1+\frac{2}{z}\right)<e^{z} E(z)<\ln \left(1+\frac{1}{z}\right) \quad \text { for } \quad z>0
$$


Gautschi's approache is dependent on a double-inequality involving the function

$$
e^{z^{p}} \int_{z}^{\infty} e^{-r^{p}} d r
$$

where $z \geq 0$ and $p>1$. By relying on a double-inequality involving the incomplete gamma function $\Gamma(v, z)$, Luke [9, p. 201] established the inequalities

$$
\begin{gathered}
\frac{z}{z+1}<z e^{z} E(z)<\frac{z+1}{z+2} \quad \text { for } \quad z>0 \\
\frac{z^{2}+3 z}{z^{2}+4 z+2}<z e^{z} E(z)<\frac{z^{2}+5 z+2}{z^{2}+6 z+6} \text { for } z>0 .
\end{gathered}
$$

Also, by depending on a double-inequality involving the function

$$
\frac{1}{\Gamma(1+1 / p)} \int_{z}^{\infty} e^{-r^{p}} d r
$$

where $z>0$ and $p \in(0,1) \cup(1, \infty)$, Alzer [2] established the inequality

$$
-\ln \left(1-e^{-a z}\right)<E(z)<-\ln \left(1-e^{-b z}\right) \quad \text { for } \quad z>0
$$

where $a \geq e^{\gamma}, 0<b \leq 1$ and $\gamma$ is the Euler-Mascheroni constant.

We observe that the bounds in (5) are bettter than those in (4). Also, the lower bound of (5) is better than that of (6) and the upper bound of $(6)$ is better than that of (4). Also, the upper bound of (6) is better than that of (5) if $z>0.756244$. Moreover, the bounds in (7) are better than those in (6). Furthermore, the lower bound of (8) is better than that of (5). However, the upper bound of (5) is better than that of (8).

By using relatively simple procedures, the objectives of this paper are:

(a) to provide two new proofs of the inequality (6),

(b) to provide a new proof of the inequality (5) by applying (6),

(c) to establish a generalization of the inequality (5),

(d) to establish new inequalities similar to (7),

(e) to establish an inequality analogous to 8 .

\section{Results and Discussion}

Theorem 2.1 ([9]). The inequality

$$
\frac{z}{z+1}<z e^{z} E(z)<\frac{z+1}{z+2}
$$

holds for $z>0$.

First Proof. Let $x>0, y>0, u>1$ and $\frac{1}{u}+\frac{1}{v}=1$. Then by using Hölder's inequality, we obtain

$$
\begin{aligned}
E\left(\frac{x}{u}+\frac{y}{v}\right) & =\int_{1}^{\infty} \frac{e^{-\left(\frac{x}{u}+\frac{y}{v}\right) r}}{r} d r \\
& =\int_{1}^{\infty} \frac{e^{-\frac{x t}{u}}}{r^{\frac{1}{u}}} \frac{e^{-\frac{y t}{v}}}{r^{\frac{1}{v}}} d r \\
& \leq\left(\int_{1}^{\infty} \frac{e^{-x r}}{r} d r\right)^{\frac{1}{u}}\left(\int_{1}^{\infty} \frac{e^{-y r}}{r} d r\right)^{\frac{1}{v}} \\
& =E^{\frac{1}{u}}(x) E^{\frac{1}{v}}(y) .
\end{aligned}
$$


Thus, $E(z)$ is logarithmically convex for $z>0$. By applying (2) and (3), we obtain

$$
\begin{aligned}
(\ln E(z))^{\prime \prime} & =\frac{E^{\prime \prime}(z) E(z)-\left[E^{\prime}(z)\right]^{2}}{E^{2}(z)} \\
& =\frac{(z+1) e^{z} E(z)-1}{e^{2 z} z^{2} E^{2}(z)} \geq 0 .
\end{aligned}
$$

Hence $(z+1) e^{z} E(z)-1>0$ which gives the left-hand side of (9). Next let

$$
\mathcal{A}(z)=E(z)-\frac{(z+1) e^{-z}}{(z+2) z} \quad \text { for } \quad z>0 .
$$

Then

$$
\begin{aligned}
\mathcal{A}^{\prime}(z) & =E^{\prime}(z)+\frac{e^{-z}}{z^{2}}\left(\frac{z^{3}+4 z^{2}+4 z+2}{z^{2}+4 z+4}\right) \\
& =\frac{e^{-z}}{z}\left(\frac{z^{3}+4 z^{2}+4 z+2}{z^{3}+4 z^{2}+4 z}-1\right)>0
\end{aligned}
$$

which implies that $\mathcal{A}(z)$ is increasing. Hence for $z>0$, we have

$$
\mathcal{A}(z)<\lim _{z \rightarrow \infty} \mathcal{A}(z)=0
$$

which gives the right-hand side of (9). This completes the proof.

Second Proof. Let $\mathcal{D}(z)=z e^{z} E(z)$ for $z>0$. It is known in [6, p. 194] that

$$
\mathcal{D}(z)=1-\int_{0}^{1} \frac{r^{z-1}}{(1-\ln r)^{2}} d r .
$$

Then

$$
\mathcal{D}^{\prime}(z)=-\int_{0}^{1} \frac{(\ln r) r^{z-1}}{(1-\ln r)^{2}} d r>0
$$

which shows that $\mathcal{D}(z)$ is increasing. Thus,

$$
\mathcal{D}^{\prime}(z)=(z+1) e^{z} E(z)-1>0
$$

which yields the left-hand side of (9). Also,

$$
\mathcal{D}^{\prime \prime}(z)=-\int_{0}^{1} \frac{(\ln r)^{2} r^{z-1}}{(1-\ln r)^{2}} d r<0
$$

which shows that $\mathcal{D}(z)$ is concave. Thus,

$$
\mathcal{D}^{\prime \prime}(z)=(z+2) e^{z} E(z)-\frac{z+1}{z}<0
$$

which yields the right-hand side of $(9$ ). This completes the proof.

Remark 2.2. It follows from (10) that,

$$
(-1)^{k} \mathcal{D}^{(k+1)}(z)=(-1)^{k+1} \int_{0}^{1} \frac{(\ln r)^{k+1} r^{z-1}}{(1-\ln r)^{2}} d r>0
$$

for all $k \in \mathbb{N}$. Therefore, $\mathcal{D}^{\prime}(z)$ is strictly completely monotonic. Also, since $\lim _{z \rightarrow \infty} \mathcal{D}(z)=1$, and by the monotonicity property of $\mathcal{D}(z)$, we recover the right-hand side of 44 . 
Theorem 2.3 ([5]). The inequality

$$
\frac{1}{2} \ln \left(1+\frac{2}{z}\right)<e^{z} E(z)<\ln \left(1+\frac{1}{z}\right)
$$

holds for $z>0$.

Proof. Let $\phi(z)=e^{z} E(z)-\ln \left(1+\frac{1}{z}\right)$ for $z>0$. By applying the left-hand side of (9), we obtain

$$
\phi^{\prime}(z)=e^{z} E(z)-\frac{1}{z+1}>0
$$

which implies that $\phi(z)$ is increasing. Consequently, we obtain

$$
-\gamma=\lim _{z \rightarrow 0} \phi(z)<\phi(z)<\lim _{z \rightarrow \infty} \phi(z)=0
$$

which gives

$$
-\gamma+\ln \left(1+\frac{1}{z}\right)<e^{z} E(z)<\ln \left(1+\frac{1}{z}\right)
$$

Likewise, let $\psi(z)=e^{z} E(z)-\frac{1}{2} \ln \left(1+\frac{2}{z}\right)$ for $z>0$. By applying the right-hand side of (9), we obtain

$$
\psi^{\prime}(z)=e^{z} E(z)-\frac{z+1}{z(z+2)}<0
$$

which implies that $\psi(z)$ is decreasing. Hence, we obtain

$$
\psi(z)>\lim _{z \rightarrow \infty} \psi(z)=0
$$

which gives

$$
e^{z} E(z)>\frac{1}{2} \ln \left(1+\frac{2}{z}\right) .
$$

Combining (13) and the upper part of 12 yields 111 . This completes the proof.

Remark 2.4. The lower bound of 12 has been discovered as a byproduct of the proof and for $0<z<$ 0.20845 , it is better than the lower bound of 11 .

In the following theorem, we provide a generalization of Theorem 2.3 .

Theorem 2.5. The inequality

$$
\frac{1}{a} \ln \left(1+\frac{a}{z}\right)<e^{z} E(z)<\frac{1}{b} \ln \left(1+\frac{b}{z}\right)
$$

holds for $z>0$ where $a \geq 2$ and $0<b \leq 1$.

Proof. Let $\mathcal{G}(z)=e^{z} E(z)-\frac{1}{a} \ln \left(1+\frac{a}{z}\right)$ for $z>0$ and $a \geq 2$. Since

$$
\frac{z+a-1}{z+a} \geq \frac{z+1}{z+2}
$$

for all $a \geq 2$, by applying right-hand side of $(9)$, we obtain

$$
\begin{aligned}
z \mathcal{G}^{\prime}(z) & =z e^{z} E(z)-\frac{z+a-1}{z+1} \\
& \leq z e^{z} E(z)-\frac{z+1}{z+2}<0 .
\end{aligned}
$$

Hence $\mathcal{G}(z)$ is decreasing. As a result, we have

$$
\mathcal{G}(z)>\lim _{z \rightarrow \infty} \mathcal{G}(z)=0
$$


which gives the left-hand side of 14 . Similarly, let $\mathcal{H}(z)=e^{z} E(z)-\frac{1}{b} \ln \left(1+\frac{b}{z}\right)$ for $z>0$ and $0<b \leq 1$. Since

$$
\frac{z+b-1}{z+b} \leq \frac{z}{z+1}
$$

for all $0<b \leq 1$, by applying left-hand side of $(9)$, we obtain

$$
\begin{aligned}
z \mathcal{H}^{\prime}(z) & =z e^{z} E(z)-\frac{z+b-1}{z+b} \\
& \geq z e^{z} E(z)-\frac{z}{z+1}>0 .
\end{aligned}
$$

Hence $\mathcal{H}(z)$ is increasing. In view of this, we have

$$
\mathcal{H}(z)<\lim _{z \rightarrow \infty} \mathcal{H}(z)=0
$$

which gives the right-hand side of (14). This completes the proof.

The following theorem is motivated by Luke's second inequality (7).

Theorem 2.6. The inequality

$$
\frac{z^{3}+2 z^{2}-z}{z^{3}+3 z^{2}}<z e^{z} E(z)<\frac{z^{3}+3 z^{2}-2 z+2}{z^{3}+4 z^{2}}
$$

holds for $z>0$.

Proof. By employing (10), we obtain

$$
\mathcal{D}^{(3)}(z)=-\frac{2 z-1}{z^{2}}+e^{z}(z+3) E(z)-1>0
$$

which when rearranged gives the left-hand side of 15 . Similarly, we have

$$
\mathcal{D}^{(4)}(z)=-\frac{3 z^{2}-2 z+2}{z^{3}}+e^{z}(z+4) E(z)-1<0
$$

which when rearranged gives the right-hand side of 15 . This completes the proof.

Remark 2.7. The bounds in (7) are better than those of (15). By using the higher derivatives of $\mathcal{D}(z)$ and their associated monotonicities, one can derive other bounds similar to (15). For example, see the following theorem.

Theorem 2.8. The inequality

$$
\frac{z^{4}+4 z^{3}-3 z^{2}+4 z-6}{z^{4}+5 z^{3}}<z e^{z} E(z)<\frac{z^{5}+5 z^{4}-4 z^{3}+6 z^{2}-12 z+24}{z^{5}+6 z^{4}}
$$

holds for $z>0$.

Proof. This follows from the derivatives $\mathcal{D}^{(5)}(z)$ and $\mathcal{D}^{(6)}(z)$ and their inherent monotonicities. This completes the proof.

Remark 2.9. The lower bound of $\sqrt{16}$ is better than that of $\sqrt{15}$ if $z>3$ and the reverse case happens if $0<z<3$. The upper bound of $(15)$ is better than that of $(16)$.

Theorem 2.10. The inequality

$$
-\gamma+\ln \alpha-\ln \left(1-e^{-\alpha z}\right)<E(z)<-\ln \left(1-e^{-\alpha z}\right)
$$

holds for $z>0$ where $0<\alpha \leq 1$. 
Proof. Let $\mathcal{K}(z)=E(z)+\ln \left(1-e^{-\alpha z}\right)$ for $z>0$ and $0<\alpha \leq 1$. Then

$$
\mathcal{K}^{\prime}(z)=-\frac{e^{-z}}{z}+\frac{\alpha e^{-\alpha z}}{1-e^{-\alpha z}}
$$

By using the basic inequality $e^{z}>1+z$ for $z \neq 0$, we obtain

$$
\frac{1}{z}<\frac{\alpha}{1-e^{-\alpha z}}
$$

and since $e^{-z} \leq e^{-\alpha z}$, we have

$$
\frac{e^{-z}}{z}<\frac{\alpha e^{-\alpha z}}{1-e^{-\alpha z}}
$$

Hence $\mathcal{K}(z)$ is increasing and subsequently, we obtain

$$
-\gamma+\ln \alpha=\lim _{z \rightarrow 0^{+}} \mathcal{K}(z)<\mathcal{K}(z)<\lim _{z \rightarrow \infty} \mathcal{K}(z)=0
$$

which gives the inequality (17). This completes the proof.

Remark 2.11. The right-hand side of (17) agrees with the right-hand side of Alzer's result (8). However, if $\alpha=1$ and $a=e^{\gamma}$, then the left-hand side of $(8)$ is better than that of (17).

\section{Acknowledgment}

The author wish to thank the anonymous reviewers and the Editor for careful reading of the paper and for their comments and suggestions which helped to improve the quality of this paper.

\section{References}

[1] Abramowitz, M. and Stegun, I. A., 1965, Handbook of mathematical functions with formulas, graphic and mathematical Tables, Dover Publications, Inc., New York.

[2] Alzer, H., 1997, On some inequalities for the incomplete gamma function, Math. Comp., 66(218), 771-778.

[3] Bhandari, P. K. and Bissu, S. K., 2016, On some inequalities involving Turan-type inequalities, Cogent Math., 3(1), Article: 1130678.

[4] Chiccoli, C., Lorenzutta, S. and Maino, G., 1990, Recent results for generalized exponential integrals, Computers Math. Applic., 19(5), 21-29.

[5] Gautschi, W., 1959, Some elementary inequalities relating to the gamma and incomplete gamma function, J. Math. Phys., 38, 77-81.

[6] Gelle, M. and Ng, E. W., 1969, A table of integrals of the exponential integral, Journal of Research of the National Bureau of Standards - B, Mathematics and Mathematical Science, 73B(3), 191-210.

[7] Hopf, E., 1934, Mathematical problems of radiative equilibrium, No. 31, Cambridge Tracts in Mathematics and Mathematical Physics, Cambridge University Press. London.

[8] Jameson, G. J. O., 2017, The real exponential integrals, Available online at: https://www.maths. lancs.ac.uk/ jameson/expint.pdf.

[9] Luke, Y. L., 1969, The special functions and their approximations, Vol. 2, Academic Press, New York.

[10] Masina, E. 2019, A review on the exponential-integral special function and other strictly related special functions, arXiv:190\%.12373v1 [math.GM]. 
[11] Nantomah, K., 2021, A harmonic mean inequality for the exponential integral function, Int. J. Appl. Math., 34(4), 647-652.

[12] Nantomah, K., 2021, A harmonic mean inequality concerning the generalized exponential integral function, Adv. Math. Sci. J., 10(9), 3227-3231.

[13] Nantomah, K., Merovci, F. and Nasiru, S., 2017, A Generalization of the exponential integral and some associated inequalities, Honam Mathematical J., 39(1), 49-59.

[14] Olver, F. W. J., Lozier, D. W., Boisvert, R. F. and Clark, C. W., 2010, (eds) NIST handbook of mathematical functions, Cambridge University Press, London.

[15] Salem, A., 2013, A q-analogue of the exponential integral, Afr. Mat., 24, 117-125.

[16] Sroysang, B., 2013, On the n-th derivative of the exponential integral functions, Communications in Mathematics and Applications, 4(2), 141-144.

[17] Sulaiman, W. T., 2012, Turan inequalities for the exponential integral functions, Commun. Optim. Theory, 1(1), 35-41.

[18] Yakubu, A., Nantomah, K. and Iddrisu, M. M., 2020, A p-analogue of the exponential integral function and some properties, Adv. Inequal. Appl., 2020, Article ID 7. 